PROCEEDINGS OF THE AMERICAN MATHEMATICAL SOCIETY

Volume 124, Number 8, August 1996

\title{
INTEGRAL INCLUSIONS OF UPPER SEMI-CONTINUOUS OR LOWER SEMI-CONTINUOUS TYPE
}

\author{
DONAL O'REGAN
}

(Communicated by Palle E. T. Jorgensen)

\begin{abstract}
Topological results for set valued maps are used to establish existence results for integral inclusions of Volterra or Hammerstein type.
\end{abstract}

\section{INTRODUCTION}

This paper presents existence results for Volterra integral inclusions of the form

$$
y(t) \in \int_{0}^{t} k(t, s) F(s, y(s)) d s+g(t), \quad t \in[0, T],
$$

and Hammerstein integral inclusions of the form

$$
y(t) \in \int_{0}^{T} k(t, s) F(s, y(s)) d s+g(t), \quad t \in[0, T],
$$

where $F:[0, T] \times \mathbf{R} \rightarrow \mathbf{R}$ is a multivalued function with nonempty compact values. Here $T>0$ is a constant. Throughout this paper the map $x \mapsto F(t, x)$ is either upper semi-continuous or lower semi-continuous for a.e. $t \in[0, T]$.

The study of integral equations has received much attention over the last fifty years or so. However very few results are available for integral inclusions; see [2]; [3, chapter 4]; [7, chapter 12] and their references. Our paper has two main objectives. We first show that the techniques applied in establishing existence results for integral equations $[3,4,7,9,10]$ transfer naturally to integral inclusions. Our ideas were motivated by results of Pruszko [11,12] (for second order differential inclusions) and more recently by results of Frigon and Granas [6]. Our second objective is to use these ideas to establish existence results for superlinear integral inclusions; these results are new even for integral equations.

The paper will be divided into three main sections. Section 2 gathers together some known results on multivalued maps. We will then use these results in section 3 to establish existence principles for the integral inclusions (1.1) and (1.2). Existence theory for sublinear and superlinear integral inclusions will be established in section 4 .

Received by the editors February 6, 1995.

1991 Mathematics Subject Classification. Primary 45D05, 45G10.

(C)1996 American Mathematical Society 


\section{Preliminaries}

Let $E_{1}, E_{2}$ be two Banach spaces, $X$ a nonempty closed subset of $E_{1}$ and $S$ a measurable space (respectively $S=I \times \mathbf{R}^{n}$, where $I$ is a real interval, and $A \subseteq S$ is $\mathcal{L} \otimes \mathcal{B}$ measurable if $A$ belongs to the $\sigma$-algebra generated by all sets of the form $N \times D$ where $N$ is Lebesgue measurable in $I$ and $D$ is Borel measurable in $\mathbf{R}^{n}$ ). Let $H: X \rightarrow E_{2}$ and $G: S \rightarrow E_{2}$ be two multifunctions with nonempty closed values. The function $G$ is measurable (respectively $\mathcal{L} \otimes \mathcal{B}$ measurable) if the set $\{t \in S: G(t) \cap B \neq \varnothing\}$ is measurable for any closed set $B$ in $S$. A subset $A$ of $L^{1}\left([0, T], \mathbf{R}^{n}\right)$ is decomposable if for all $u, v \in A$ and $N \subseteq[0, T]$ measurable the function $u \chi_{N}+v \chi_{[0, T] / N} \in A$.

The function $H$ is lower semi-continuous (l.s.c.) (respectively upper semi-continuous (u.s.c.)) if the set $\{x \in X: H(x) \cap B \neq \varnothing\}$ is open (respectively closed) for any open (respectively closed) set $B$ in $E_{2}$. If $H$ is l.s.c. and u.s.c., then $H$ is continuous. $H$ is compact if $\overline{H(X)}=\overline{\bigcup_{x \in X} H(x)}$ is compact in $E_{2}$ and it is completely continuous if $\overline{H(\Omega)}$ is compact for all bounded sets $\Omega \subseteq X$.

$H$ is called weakly upper semi-continuous (w-u.s.c.) if for all $\left\{x_{n}\right\} \subseteq X$ and $\left\{y_{n}\right\} \subseteq E_{2}$ the condition

$$
x_{n} \rightarrow x, y_{n} \rightarrow y, y_{n} \in H\left(x_{n}\right) \text { for all } n
$$

implies $y \in H(x)$.

Remark. Here $\rightarrow$ denotes weak convergence.

$H$ is called weakly completely continuous if $H$ is $w$-u.s.c. and for every bounded subset $\Omega$ of $X, H(\Omega)=\bigcup_{a \in \Omega} H(a)$ is a relatively weakly compact subset of $E_{2}$.

We now state without proof (see $[11,12])$ two results.

Theorem 2.1. Let $H: X \rightarrow E_{2}$ be a completely continuous multivalued map. Then $H$ is u.s.c. if and only if the graph $\mathcal{G}(H)=\left\{(x, y) \in X \times E_{2}: y \in H(x)\right\}$ is a closed subset of $X \times E_{2}$.

Theorem 2.2. Let $E$ be any Banach space. Suppose $H: X \rightarrow E_{2}$ is a weakly completely continuous multivalued mapping and $T: E_{2} \rightarrow E$ is given by $T(x)=$ $T_{1}(x)+p, x \in E_{2}$, where $p$ is a (fixed) element of $E$ and $T_{1}: E_{2} \rightarrow E$ is a continuous linear mapping. Then the graph $\mathcal{G}(T \circ H)$ of the mapping $T \circ H: X \rightarrow E$ is a closed subset of $X \times E$.

Remark. The proof of Theorem 2.2 is immediate from the above definitions, see also [11, Proposition 1.5].

Let $F:[0, T] \times \mathbf{R}^{n} \rightarrow \mathbf{R}^{n}$ be a multivalued function with nonempty compact values. Throughout this paper our map $F$ will satisfy some of the following properties (to be specified later):

$$
\left\{\begin{array}{l}
\text { (i) }(t, x) \mapsto F(t, x) \text { is } \mathcal{L} \otimes \mathcal{B} \text { measurable, } \\
\text { (ii) } x \mapsto F(t, x) \text { is l.s.c. for a.e. } t \in[0, T],
\end{array}\right.
$$

$\left\{\begin{array}{l}\text { (i) } t \mapsto F(t, x) \text { is measurable for every } x \in \mathbf{R}^{n}, \\ \text { (ii) } x \mapsto F(t, x) \text { is continuous for a.e. } t \in[0, T],\end{array}\right.$

$\left\{\begin{array}{l}\text { (i) } t \mapsto F(t, x) \text { is measurable for every } x \in \mathbf{R}^{n}, \\ \text { (ii) } x \mapsto F(t, x) \text { is u.s.c. for a.e. } t \in[0, T],\end{array}\right.$ 


$$
\left\{\begin{array}{l}
\text { for each } r>0 \text { there exists a function } h_{r} \in L^{1}[0, T] \text { such that } \\
|F(t, x)| \leq h_{r}(t) \text { for a.e. } t \in(0, T) \text { and every } x \in \mathbf{R}^{n} \text { with }|x| \leq r .
\end{array}\right.
$$

Assign to $F$ a multivalued operator

$$
\mathcal{F}: C[0, T] \rightarrow L^{1}[0, T]
$$

by letting

$$
\mathcal{F}(y)=\left\{w \in L^{1}[0, T]: w(t) \in F(t, y(t)) \text { for a.e. } t \in(0, T)\right\} .
$$

Theorem 2.3 ([11]). Let $F:[0, T] \times \mathbf{R}^{n} \rightarrow \mathbf{R}^{n}$ satisfy (2.3) and (2.4). Then the multivalued mapping $\mathcal{F}: C[0, T] \rightarrow L^{1}[0, T]$ defined by (2.5) is weakly completely continuous and integrably bounded on bounded sets.

Remark. $\mathcal{F}$ is integrably bounded on bounded sets if for every bounded set $A$ there exists $h_{A} \in L^{1}[0, T]$ such that for every $x \in A$ and $w \in \mathcal{F}(x)$ we have $|w(t)| \leq h_{A}(t)$ a.e. on $(0, T)$.

Next we state a recent selection theorem [1] due to Bressan and Colombo. Let $Y$ be a metric space and $G: Y \rightarrow L^{1}[0, T]$ a multivalued operator. We say $G$ has property $(B C)$ if

(i) $G$ is l.s.c.

(ii) $G$ has nonempty closed and decomposable values.

Theorem 2.4 ([1]). Let $Y$ be a separable metric space and let $G: Y \rightarrow L^{1}[0, T]$ be a multivalued operator which has property $(B C)$. Then $G$ has a continuous selection, i.e. there exists a continuous function (single valued) $g: Y \rightarrow L^{1}[0, T]$ such that $g(y) \in G(y)$ for every $y \in Y$.

Finally we state the two fixed point results which will be used in section 3 .

Theorem 2.5 (Nonlinear alternative for single valued maps [5, 8]). Assume $U$ is a relatively open subset of a convex set $K$ in a Banach space $E$. Let $N: \bar{U} \rightarrow K$ be a compact map with $p \in U$. Then either

(i) $N$ has a fixed point in $\bar{U}$; or

(ii) there is $a u \in \partial U$ and $a \lambda \in(0,1)$ such that $u=\lambda N u+(1-\lambda) p$.

Theorem 2.6 (Nonlinear alternative for multivalued maps [5]). Let $K$ be a convex subset of a Banach space, $U \subseteq K$ be relatively open, and $p \in U$. Suppose $N: \bar{U} \rightarrow K$ is an u.s.c. compact multivalued map with nonempty, compact, convex values. Then either

(i) there is a $u \in \bar{U}$ such that $u \in N u$; or

(ii) there is a $u \in \partial U$ and $a \lambda \in(0,1)$ such that $u \in \lambda N u+(1-\lambda) p$.

\section{EXISTENCE PRINCIPLES}

We begin by presenting some existence principles for the Volterra integral inclusion

$$
y(t) \in \int_{0}^{t} k(t, s) F(s, y(s)) d s+g(t), \quad t \in[0, T] .
$$

Now $F:[0, T] \times \mathbf{R} \rightarrow \mathbf{R}$ is a multivalued function with nonempty compact values. The kernel $k$ satisfies the following conditions:

$$
\left\{\begin{array}{l}
\text { for each } t \in[0, T], k(t, s) \text { is measurable on }[0, t] \text { and } \\
k(t)=\operatorname{ess} \sup |k(t, s)|, 0 \leq s \leq t, \text { is bounded on }[0, T]
\end{array}\right.
$$




$$
\left\{\begin{array}{l}
\text { the map } t \mapsto k_{t} \text { is continuous from }[0, T] \text { to } L^{\infty}[0, T] ; \\
\text { here } k_{t}(s)=k(t, s) .
\end{array}\right.
$$

Now assume $g:[0, T] \rightarrow \mathbf{R}$ is single valued with

$$
g \in C[0, T] .
$$

Define the operator

$$
S: L^{1}[0, T] \rightarrow C[0, T]
$$

by

$$
S h(t)=\int_{0}^{t} k(t, s) h(s) d s+g(t) .
$$

To see that the above makes sense let $h \in L^{1}[0, T]$. Notice first that

$$
\left|\int_{0}^{t} k(t, s) h(s) d s\right| \leq\left(\sup _{[0, T]} k(t)\right) \int_{0}^{T}|h(s)| d s .
$$

Also for $t, \tau \in[0, T]$ with $t<\tau$ we have

$$
\begin{aligned}
|S h(t)-S h(\tau)| & =\left|\int_{0}^{t} k(t, s) h(s) d s-\int_{0}^{\tau} k(\tau, s) h(s) d s\right| \\
& =\left|\int_{0}^{\tau}[k(t, s)-k(\tau, s)] h(s) d s+\int_{\tau}^{t} k(t, s) h(s) d s\right| \\
& \leq|k(t, \cdot)-k(\tau, \cdot)|_{L^{\infty}} \int_{0}^{T}|h(s)| d s+\left(\sup _{[0, T]} k(t)\right) \int_{t}^{\tau}|h(s)| d s .
\end{aligned}
$$

Lemma 3.1. Suppose (3.2), (3.3) and (3.4) are satisfied. Then the operator $S$ : $L^{1}[0, T] \rightarrow C[0, T]$, given by (3.6), is continuous.

Proof. Let $h_{1}, h_{2} \in L^{1}[0, T]$. Then

$$
\begin{aligned}
\left|S h_{1}(t)-S h_{2}(t)\right| & =\left|\int_{0}^{t} k(t, s)\left[h_{1}(s)-h_{2}(s)\right] d s\right| \\
& \leq\left(\sup _{[0, T]} k(t)\right) \int_{0}^{T}\left|h_{1}(s)-h_{2}(s)\right| d s .
\end{aligned}
$$

Define the multivalued operator

$$
\mathcal{F}: C[0, T] \rightarrow L^{1}[0, T]
$$

by

$$
\mathcal{F}(y)=\left\{w \in L^{1}[0, T]: w(t) \in F(t, y(t)) \text { for a.e. } t \in(0, T)\right\} .
$$

Definition. Let $F:[0, T] \times \mathbf{R} \rightarrow \mathbf{R}$ be a multivalued function with nonempty compact values. We say

(i) $F$ is of lower semi-continuous type (l.s.c. type) if $\mathcal{F}$, given by (3.7), is l.s.c. and has nonempty, closed and decomposable values.

(ii) $S \circ F$ is of upper semi-continuous type (u.s.c. type) if $S \circ \mathcal{F}$, given in (3.6) and (3.7), is u.s.c., completely continuous and has nonempty, compact, convex values. 
Theorem 3.2. Let $F:[0, T] \times \mathbf{R} \rightarrow \mathbf{R}$ be a multivalued function with nonempty, compact, convex values. Assume (2.3), (2.4), (3.2), (3.3) and (3.4) are satisfied. Then $S \circ F$ is of u.s.c. type.

Proof. Theorem 2.3 implies that $\mathcal{F}: C[0, T] \rightarrow L^{1}[0, T]$ is weakly completely continuous and integrably bounded on bounded sets. Notice for any $v \in L^{1}[0, T]$ that $S v(t)=S v_{1}(t)+g(t)$ where $S_{1}: L^{1}[0, T] \rightarrow C[0, T]$ is the continuous linear operator given by

$$
S_{1} v(t)=\int_{0}^{t} k(t, s) v(s) d s .
$$

Now Theorem 2.2 implies that $\mathcal{G}(S \circ \mathcal{F})$ is a closed subset of $C[0, T] \times L^{1}[0, T]$. In addition notice if $\Omega$ is any bounded subset of $C[0, T]$, then $S \circ \mathcal{F}(\Omega)$ is relatively compact. Now Theorem 2.1 implies that $S \circ \mathcal{F}$ is u.s.c.

Theorem $3.3([6])$. Let $F:[0, T] \times \mathbf{R} \rightarrow \mathbf{R}$ be a multivalued function with nonempty, compact values. Assume (2.4) holds with F satisfying either (2.1) or (2.2). Then $F$ is of l.s.c. type.

We now prove two existence principles for the integral inclusion (3.1).

Theorem 3.4. Let $F:[0, T] \times \mathbf{R} \rightarrow \mathbf{R}$ be a multivalued function with nonempty, compact, convex values. Suppose (2.3), (2.4), (3.2), (3.3) and (3.4) are satisfied. In addition assume there is a constant $M_{0}$, independent of $\lambda$, with

$$
|y|_{0}=\sup _{[0, T]}|y(t)| \neq M_{0}
$$

for any solution y to

$$
y(t) \in \lambda\left(\int_{0}^{t} k(t, s) F(s, y(s)) d s+g(t)\right), \quad t \in[0, T],
$$

for each $\lambda \in(0,1)$. Then (3.1) has a solution in $C[0, T]$.

Proof. Solving $(3.8)_{\lambda}$ is equivalent to the fixed point problem $y \in S \circ \mathcal{F}(y) \equiv \lambda N y$ where $N=S \circ \mathcal{F}$ (here $S$ is as in (3.6) and $\mathcal{F}$ is as in (3.7)). Notice from Theorem 3.2 that $N$ is of u.s.c. type. Let

$$
U=\left\{u \in C[0, T]:|u|_{0}<M_{0}\right\} \quad \text { and } \quad E=K=C[0, T] .
$$

Apply Theorem 2.6 (nonlinear alternative for multivalued maps) and we may deduce the result immediately.

Theorem 3.5. Let $F:[0, T] \times \mathbf{R} \rightarrow \mathbf{R}$ be a multivalued function with nonempty, compact values. Suppose (2.4), (3.2), (3.3) and (3.4) are satisfied. Also assume F satisfies either (2.1) or (2.2). In addition suppose there is a constant $M_{0}$, independent of $\lambda$, with $|y|_{0} \neq M_{0}$ for any solution $y$ to $(3.8)_{\lambda}$ for each $\lambda \in(0,1)$. Then (3.1) has a solution in $C[0, T]$.

Proof. Solving $(3.8)_{\lambda}$ is equivalent to the fixed point problem $y \in \lambda N_{1} y$ where $N_{1}=S \circ \mathcal{F}$. Now Theorem 3.3 together with the Bressan Colombo selection theorem (Theorem 2.4) implies that $F$ has a continuous selection $f: C[0, T] \rightarrow L^{1}[0, T]$. Consider the family of problems

$$
y(t)=\lambda\left(\int_{0}^{t} k(t, s) f(s, y(s)) d s+g(t)\right), \quad t \in[0, T],
$$


for $\lambda \in(0,1)$. By assumption, any solution $y$ to $(3.9)_{\lambda}$ satisfies $|y|_{0} \neq M_{0}$. Let

$$
U=\left\{u \in C[0, T]:|u|_{0}<M_{0}\right\} \quad \text { and } \quad E=K=C[0, T]
$$

and

$$
N: C[0, T] \rightarrow C[0, T] \text { is defined by } N y(t)=\int_{0}^{t} k(t, s) f(s, y(s)) d s+g(t) .
$$

Remark. Notice $N$ is continuous and completely continuous (follows immediately from the Arzela-Ascoli theorem and the analogue of the two displayed equations after (3.6)).

Apply Theorem 2.5 (nonlinear alternative for single valued maps) to deduce that $(3.9)_{1}$ has a solution. Consequently (3.1) has a solution.

Next we present two existence principles for the Hammerstein integral inclusion

$$
y(t) \in \int_{0}^{T} k(t, s) F(s, y(s)) d s+g(t), \quad t \in[0, T] .
$$

Here $F:[0, T] \times \mathbf{R} \rightarrow \mathbf{R}$ is a multivalued function with nonempty compact values and $g:[0, T] \rightarrow \mathbf{R}$ is a single valued function. The kernel $k$ satisfies

$$
\left\{\begin{array}{l}
\text { for each } t \in[0, T], k(t, s) \text { is measurable on }[0, T] \text { and } \\
k(t)=\operatorname{ess} \sup |k(t, s)|, 0 \leq s \leq T, \text { is bounded on }[0, T] .
\end{array}\right.
$$

Essentially the same reasoning as in Theorems 3.4 and 3.5 establishes the following two existence principles for the integral inclusion (3.10).

Theorem 3.6. Let $F:[0, T] \times \mathbf{R} \rightarrow \mathbf{R}$ be a multivalued function with nonempty, compact, convex values. Suppose (2.3), (2.4), (3.3), (3.4) and (3.11) are satisfied. In addition assume there is a constant $M_{0}$, independent of $\lambda$, with $|y|_{0} \neq M_{0}$ for any solution $y$ to

$$
y(t) \in \lambda\left(\int_{0}^{T} k(t, s) F(s, y(s)) d s+g(t)\right), \quad t \in[0, T],
$$

for each $\lambda \in(0,1)$. Then $(3.10)$ has a solution in $C[0, T]$.

Theorem 3.7. Let $F:[0, T] \times \mathbf{R} \rightarrow \mathbf{R}$ be a multivalued function with nonempty, compact values. Suppose (2.4), (3.3), (3.4) and (3.11) are satisfied. Also assume F satisfies either (2.1) or (2.2). In addition suppose there is a constant $M_{0}$, independent of $\lambda$, with $|y|_{0} \neq M_{0}$ for any solution $y$ to $(3.12)_{\lambda}$ for each $\lambda \in(0,1)$. Then (3.10) has a solution in $C[0, T]$.

\section{EXISTENCE THEORY}

The usual existence results for integral equations of Volterra or Hammerstein type $[3,4,9,10]$ are easily extended (by essentially the same reasoning) to integral inclusions; consequently we will omit these results. Instead we now establish a result (which is new even in the single valued case) for the Hammerstein integral inclusion

$$
y(t) \in \mu \int_{0}^{T} k(t, s) F(s, y(s)) d s+g(t), \quad t \in[0, T],
$$

where $\mu \geq 0$ is a parameter. 
Theorem 4.1. Let $F:[0, T] \times \mathbf{R} \rightarrow \mathbf{R}$ be a multivalued function with nonempty, compact, convex values. Suppose (2.3), (2.4), (3.3), (3.4) and (3.11) are satisfied. In addition assume

$$
\left\{\begin{array}{l}
|F(t, y)| \leq q(t) f(|y|) \text { for almost all } t \in[0, T] \text { and all } y \in \mathbf{R}^{n} ; \text { here } \\
f:[0, \infty) \rightarrow[0, \infty) \text { is a Borel measurable function and } q \in L^{1}[0, T] .
\end{array}\right.
$$

Case (a): Suppose

$$
q \text { is bounded on }[0, T]
$$

and

$$
\left\{\begin{array}{l}
\text { there exists a continuous function } f_{1}:[0, \infty) \rightarrow[0, \infty) \text { such that } \\
f_{1}(u)>0 \text { for } u>0 \text { and } \int_{0}^{T} f(|u(s)|) d s \leq f_{1}\left(|u|_{0}\right) \text { for any } \\
u \in C[0, T] ; \text { here }|u|_{0}=\sup _{[0, T]}|u(t)|
\end{array}\right.
$$

hold. Let $\mu_{0}$ satisfy

$$
\sup _{c \in[0, \infty)}\left(\frac{c}{\sup _{[0, T]}|g(t)|+\mu_{0} f_{1}(c)\left(\sup _{[0, T]} k(t)\right)\left(\sup _{[0, T]} q(t)\right)}\right)>1
$$

where $k(t)=\operatorname{ess} \sup |k(t, s)|, 0 \leq s \leq T$. If $0 \leq \mu \leq \mu_{0}$, then (4.1) has a solution.

Case (b): Suppose

$$
f \text { in }(4.2) \text { is continuous and nondecreasing on }[0, \infty) \text { with } f(u)>0 \text { for } u>0
$$

is satisfied. Let $\mu_{0}$ satisfy

$$
\sup _{c \in[0, \infty)}\left(\frac{c}{\sup _{[0, T]}|g(t)|+\mu_{0} f(c)\left(\sup _{[0, T]} k(t)\right) \int_{0}^{T} q(s) d s}\right)>1 .
$$

If $0 \leq \mu \leq \mu_{0}$, then (4.1) has a solution.

Remark. The supremum in (4.5), (4.7) is allowed to be infinite.

Proof. Case (a): Fix $\mu \leq \mu_{0}$. Let $M_{0}>0$ satisfy

$$
\frac{M_{0}}{\sup _{[0, T]}|g(t)|+\mu f_{1}\left(M_{0}\right)\left(\sup _{[0, T]} k(t)\right)\left(\sup _{[0, T]} q(t)\right)}>1 \text {. }
$$

Let $y \in C[0, T]$ be a solution of

$$
y(t) \in \lambda\left(\mu \int_{0}^{T} k(t, s) F(s, y(s)) d s+g(t)\right), \quad t \in[0, T],
$$

with $\lambda \in(0,1)$. Then $y(t)=\lambda\left(\mu \int_{0}^{T} k(t, s) v(s) d s+g(t)\right)$ where $v \in F(s, y)$. Consequently for $t \in[0, T]$ we have

$$
\begin{aligned}
|y(t)| & \leq \mu k(t)\left(\sup _{[0, T]} q(t)\right) \int_{0}^{T} f(|y(s)|) d s+|g(t)| \\
& \leq \mu\left(\sup _{[0, T]} k(t)\right)\left(\sup _{[0, T]} q(t)\right) f_{1}\left(|y|_{0}\right)+\sup _{[0, T]}|g(t)| .
\end{aligned}
$$


Thus

$$
\frac{|y|_{0}}{\sup _{[0, T]}|g(t)|+\mu f_{1}\left(|y|_{0}\right)\left(\sup _{[0, T]} k(t)\right)\left(\sup _{[0, T]} q(t)\right)} \leq 1 .
$$

Suppose there exists $\lambda \in(0,1)$ with $|y|_{0}=M_{0}$. Then (4.10) implies

$$
\frac{M_{0}}{\sup _{[0, T]}|g(t)|+\mu f_{1}\left(M_{0}\right)\left(\sup _{[0, T]} k(t)\right)\left(\sup _{[0, T]} q(t)\right)} \leq 1
$$

which contradicts (4.8). Thus any solution $y$ to $(4.9)_{\lambda}$ satisfies $|y|_{0} \neq M_{0}$. Theorem 3.6 implies that (4.1) has a solution $y \in C[0, T]$.

Case (b): Fix $\mu \leq \mu_{0}$. Let $M_{0}>0$ satisfy

$$
\frac{M_{0}}{\sup _{[0, T]}|g(t)|+\mu f\left(M_{0}\right)\left(\sup _{[0, T]} k(t)\right) \int_{0}^{T} q(s) d s}>1 .
$$

Let $y$ be any solution of $(4.9)_{\lambda}$. Then for $t \in[0, T]$ we have

$$
\begin{aligned}
|y(t)| & \leq \mu k(t) \int_{0}^{T} q(s) f(|y(s)|) d s+|g(t)| \\
& \leq \mu k(t) f\left(|y|_{0}\right) \int_{0}^{T} q(s) d s+\sup _{[0, T]}|g(t)| \\
& \leq \mu f\left(|y|_{0}\right)\left(\sup _{[0, T]} k(t)\right) \int_{0}^{T} q(s) d s+\sup _{[0, T]}|g(t)|
\end{aligned}
$$

and so

$$
\frac{|y|_{0}}{\sup _{[0, T]}|g(t)|+\mu f\left(|y|_{0}\right)\left(\sup _{[0, T]} k(t)\right) \int_{0}^{T} q(s) d s} \leq 1
$$

Suppose there exists $\lambda \in(0,1)$ with $|y|_{0}=M_{0}$. Then (4.12) implies

$$
\frac{M_{0}}{\sup _{[0, T]}|g(t)|+\mu f\left(M_{0}\right)\left(\sup _{[0, T]} k(t)\right) \int_{0}^{T} q(s) d s} \leq 1
$$

which contradicts (4.11).

Remarks. (i) Notice in the proof of Theorem 4.1 we only showed that any solution of $(4.9)_{\lambda}$ satisfies $|y|_{0} \neq M_{0}$. We do not claim (and indeed it is not true in general) that any solution of $(4.9)_{\lambda}$ satisfies the inequality $|y|_{0} \leq M_{0}$. by

(ii) There is an obvious analogue of Theorem 4.1 if (4.3) and (4.4) are replaced

$$
\left\{\begin{array}{l}
\text { there exists } p>1 \text { with } q \in L^{p}[0, T] \text {. Also there exists a continuous } \\
\text { function } f_{1}:[0, \infty) \rightarrow[0, \infty) \text { such that } f_{1}(u)>0 \text { for } u>0 \text { and } \\
\left(\int_{0}^{T} f^{r}(|u(s)|) d s\right)^{\frac{1}{r}} \leq f_{1}\left(|u|_{0}\right) \text { for any } u \in C[0, T] ; \text { here } \frac{1}{p}+\frac{1}{r}=1 .
\end{array}\right.
$$

Essentially the same reasoning as in Theorem 4.1, except we use Theorem 3.7 instead of Theorem 3.6, establishes the following result.

Theorem 4.2. Let $F:[0, T] \times \mathbf{R} \rightarrow \mathbf{R}$ be a multivalued function with nonempty, compact values. Suppose (2.4), (3.3), (3.4), (3.11) and (4.2) are satisfied. Also assume $F$ satisfies either (2.1) or (2.2).

Case (a): Suppose (4.3) and (4.4) hold. Let $\mu_{0}$ satisfy (4.5). If $0 \leq \mu \leq \mu_{0}$, then (4.1) has a solution. 
Case (b): Suppose (4.6) is satisfied. Let $\mu_{0}$ satisfy (4.7). If $0 \leq \mu \leq \mu_{0}$, then (4.1) has a solution.

\section{REFERENCES}

1. A. Bressan and G. Colombo, Extensions and selections of maps with decomposable values, Studia Math. 90 (1988), 70-85. MR 89j:54021

2. A. I. Bulgakov and L. N. Lyapin, Some properties of the set of solutions for the VolterraHammerstein integral inclusion, Differential Equations (Transl.) 14 (1978), 1043-1048. MR 80a: 45007

3. C. Corduneanu, Integral equations and applications, Cambridge Univ. Press, New York, 1991. MR 92h: 45001

4. _ Perturbations of linear abstract Volterra equations, J. Internat. Eq. Appl. 2 (1990), 393-401. MR 92a:45005

5. J. Dugundji and A. Granas, Fixed point theory, Monografie Mathematyczne, PWN, Warsaw, 1982. MR 83j:54038

6. M. Frigon and A. Granas, Théorèmes d'existence pour des inclusions différentielles sans convexité, C.R. Acad. Sci. Paris, Ser. I 310 (1990), 819-822. MR 91e:34014

7. G. Gripenberg, S. O. Londen, and O. Staffans, Volterra integral and functional equations, Cambridge Univ. Press, New York, 1990. MR 91c:45003

8. D. O'Regan, Theory of singular boundary value problems, World Scientific Press, Singapore, 1994. MR 95g:34003

9. __ Existence results for nonlinear integral equations, J. Math. Anal. Appl. 192 (1995), 705-726. CMP 95:14

10. _ Existence theory for nonlinear Volterra and Hammerstein integral equations, Dynamical Systems and Applications (R. P. Agarwal, ed.), World Scientific Series in Applicable Analysis, Vol. 4 (1995), 601-615.

11. T. Pruszko, Topological degree methods in multivalued boundary value problems, J. Nonlinear Anal. 5 (1981), 953-973.

12. _ Some applications of the topological degree theory to multivalued boundary value problems, Dissertationes Math. 229 (1984). MR 85j:34030

Department of Mathematics, University College Galway, Galway, Ireland

E-mail address: donal.oregan@ucg.ie 